\title{
Multi-sensor comparison of aerosol optical properties profiles observed during a pollution episode in a mega city of the North China Plain
}

\author{
Lina $\mathrm{Gao}^{1}$, Yong Zhang ${ }^{1, *}$, Yubao Chen ${ }^{1}$, Zhichao Bu${ }^{1}$, Xinghong Cheng ${ }^{2}$, Junshan Jing ${ }^{1}$, Shanshan Lv ${ }^{1}$ \\ ${ }^{1}$ Meteorological Observation Center, China Meteorological Administration, 100081, Beijing, China \\ ${ }^{2}$ State Key Lab of Severe Weather, Key Laboratory for Atmospheric Chemistry, Chinese Academy of Meteorological Science, Beijing \\ 100081, China
}

\begin{abstract}
A fine aerosol particle pollution episode happened in mega city Beijing during February 25th March 5th. Aerosol extinction coefficient profiles were observed at 7 Lidar stations. Different aerosol optical property instruments were used to verify Lidar retrievals. Ground visibility observations were used to verify near ground Lidar observations. High correlation coefficient was found (0.8) between visiometer and Lidar observations. Lidar's Column integrated aerosol extinction coefficient was also verified by Himawari- 8 aerosol optical depth (AOD) data. The correlation coefficient between two AOD retrievals was 0.4. And the mean bias was -0.17 . CALIPSO aerosol property profiles was also used to verify Lidar retrievals. Lidar retrievals was inconsistent with CALIPSO in the boundary layer. Overall, the Lidar retrievals are consistent with other optical property sensors. And the retrials could be used for observation research and model validation.
\end{abstract}

\section{Introduction}

In order to obtain better understanding of the vertical structure of the atmosphere, field experiment about "five profiles" was conducted in mega city Beijing, including temperature, humidity, wind, water content, and aerosol. Temperature and humidity profiles obtained in the field observations have been used in data assimilation and typhoon forecast [1]. It is necessary to compare Lidar retrieved aerosol optical property with those monitored by other commonly used instruments before it can be widely used.

The Advanced Himawari Imager (AHI) sensor onboard on the Himawari-8 provides aerosol properties with high spatial and temporal resolution [2-3]. CALIPSO was launched to study the radiation effect of clouds and aerosols [4-5]. The Cloud-Aerosol Lidar with Orthogonal Polarization (CALIOP) is one of the 3 instruments of CALIPSO. CALIOP is a two wavelength polarizationsensitive lidar (532 $\mathrm{nm}$ and $1064 \mathrm{~nm}$ ), it could provide global distribution of aerosols and clouds with very high vertical and temporal resolution [6]. Visibility observation have been changed from human observer to automatic instrument visiometer since 2013 in operational observation in China. It is possible for us to use hourly visibility observations to evaluate the near ground Lidar performance.

\section{Data and Method}

Aerosol extinction coefficient data during February 25th - March 5th, 2019 at 7 stations are obtained (locations shown in Figure 1). Vertical resolution, wavelength, and bland zone height information about the Lidar are shown in Table 1. Aerosol extinction coefficient retrieved by Mie scatter Lidar was evaluated by aerosol properties observed by different sensors. They are forward scattering visiometer, Himawari-8 satellite aerosol retrievals, and CALIPSO aerosol retrievals. Co-site meteorological stations (Met Stn) and aerosol monitor stations (Aero Stn) are also shown in Figure 1 and Table 1.

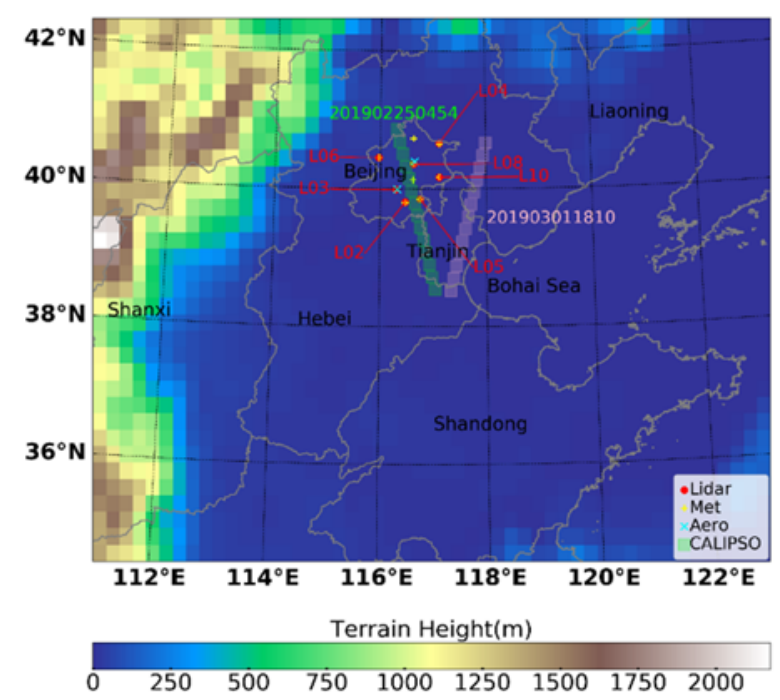


Fig. 1. Sites distribution of Lidar, Meteorological (Met), Aerosol stations and 3 trajectories of CALIPSO satellite.

Table 1. Relevant information about the Lidar Station. The wavelength of 7 lidars are all $532 \mathrm{~nm}$. H implies blind ozone height.

\begin{tabular}{|c|c|c|c|c|c|c|c|}
\hline Lidar & Stn Name & Lon $\left.\mathbf{(}^{\mathbf{9}}\right)$ & Lat $\left.\mathbf{(}^{\mathbf{}}\right)$ & Res (m) & H (m) & Met Stn & Aero Stn \\
\hline L02 & NanJiao & 39.81 & 116.47 & 7.5 & 75 & 54511 & $/$ \\
\hline L03 & Haidian & 39.99 & 116.29 & 15 & 120 & $/$ & 99012 \\
\hline L04 & Shangdianzi & 40.66 & 117.11 & 15 & 90 & 54421 & $/$ \\
\hline L05 & Tongzhou & 39.85 & 116.75 & 30 & 0 & 54431 & $/$ \\
\hline L06 & Yanqing & 40.45 & 115.97 & 3.5 & 75 & 54406 & $/$ \\
\hline L08 & Huairou & 40.36 & 116.63 & 15 & 75 & 54419 & 99014 \\
\hline L10 & Pinggu & 40.17 & 117.12 & 15 & 60 & 54424 & $/$ \\
\hline
\end{tabular}

CALIPSO data products are widely used to study the temporal and spatial variation characteristics of aerosol particles [7]. The CALIPSO Lidar Level 1.5 standard data product is selected to compare with Lidar profile in this study.

Ground aerosol extinction coefficient $\left(\sigma_{V i s}\right)$ was determined according to horizontal visibility observations at the visible light band $(550 \mathrm{~nm})$ according the Koschmieder law [8-10].

$$
\sigma_{\text {Vis }}=\frac{3000}{\text { Visibility }}
$$

where visibility is in the unit of $\mathrm{km}$, and $\sigma_{V i s}$ is in the unit of $\mathrm{Mm}^{-1}$. Considering blind zone height of Lidar, average value during $120-180 \mathrm{~m}$ were used to represent extinction coefficient on the ground.

Himawari-8 satellite retrieved aerosol optical depth (AOD) data was used to evaluate the column amount of aerosol extinction coefficient of Lidar observation. Satellite observations nearest to the Lidar stations were adopted. And aerosol optical depth of Lidar was calculated by the integral of the extinction coefficient of the whole layer. It was represented by the following equation.

$$
A O D=\sum_{\text {Level }=1}^{\text {Level }=N} \sigma_{\text {Lidar }} * \Delta H
$$

where $\sigma_{\text {Lidar }}$ was the aerosol extinction coefficient at different layers, and $\Delta \mathrm{H}$ was the layer height. $\sigma_{\text {Lidar }}$ was in the unit of $\mathrm{km}^{-1}$, and $\mathrm{H}$ was in the unit of $\mathrm{km}$.

CALIPSO backscatter coefficient retrievals was also adopted. And aerosol extinction coefficient was obtained with the Lidar constant 50.

Bias and correlation coefficient (R) of observation between Lidar and other sensors were calculated to evaluate the consistency between different sensors.

\section{Results and Discussion}

\section{1. $\mathrm{PM}_{2.5}$ mass concentration variation}

A $\mathrm{PM}_{2.5}$ pollution episode happened in Beijing during February 25 - March 5, 2019, as it is shown in Figure 2.
$\mathrm{PM}_{2.5}$ mass concentration increased from less than 100 $\mu \mathrm{g} / \mathrm{m}^{3}$ to around $200 \mu \mathrm{g} / \mathrm{m}^{3}$ at March 1 , 2019. $\mathrm{PM}_{10}$ was a little higher than $\mathrm{PM}_{2.5}$ during the episode. This implies that it was a fine particle pollution process.
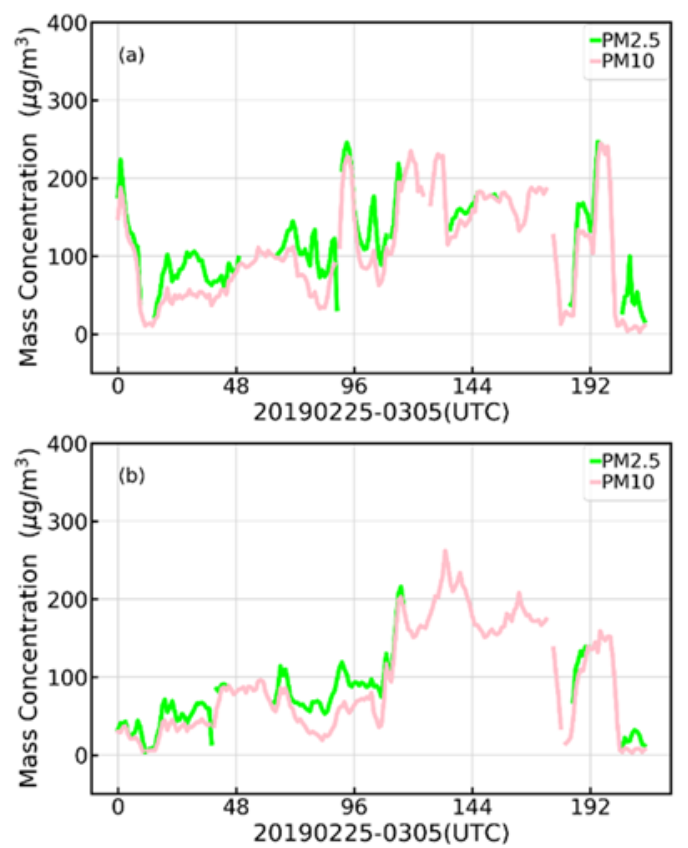

Fig. 2. Temporal variations of $\mathrm{PM}_{2.5}$ and $\mathrm{PM}_{10}$ during February 25 - March 5, 2019 in Beijing.

Vertical distribution of aerosol extinction coefficient at seven Lidar stations are shown in Figure 3. Three Lidar stations (L02, L04, and L08) have maximum extinction coefficient value less than $400 \mathrm{Mm}^{-1}$. Other 4 stations have maximum value in the range $400-600 \mathrm{Mm}^{-1}$. L8 has an average aerosol layer lift off the ground. Seven Lidar stations have similar vertical structures. 

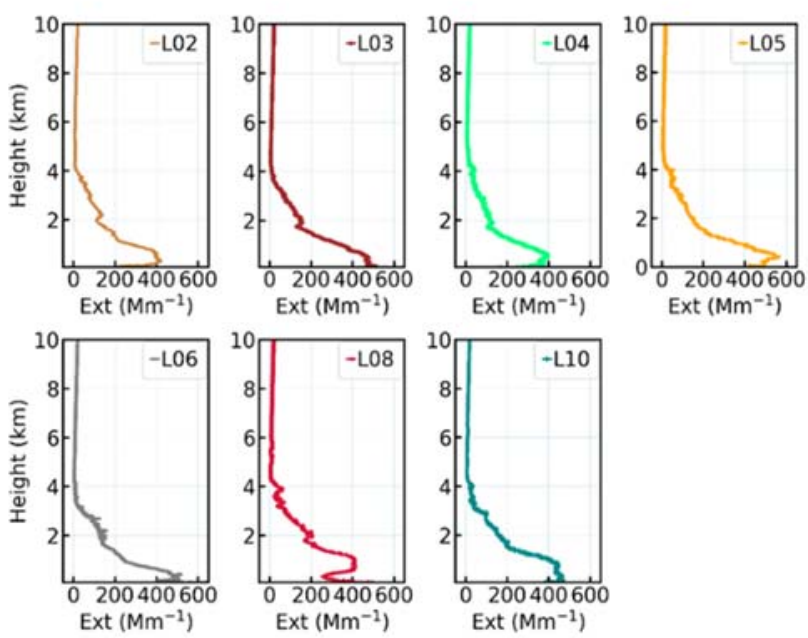

Fig. 3. Vertical distribution of aerosol extinction coefficient (Ext) at 7 lidar stations. The minimum level starts from the top of bland zone of each Lidar.

\subsection{Lidar and ground observations}

Ground extinction coefficient observations at 6 stations were used to evaluate near ground Lidar retrievals (Figure 4 and Table 2). Both sensors show the trend of low first and then high pollution episode. Extinction coefficient retrieved by Lidar was lower than ground visiometers. The mean bias between Lidar observations and visiometer observations was $342.50 \mathrm{Mm}^{-1}$. It indicates that visiometer observations were higher than Lidar retrievals. But L06 retrievals were higher than ground visiometer observations. The mean correlation coefficient was 0.8 . L06 had the highest value 0.91. Considering wavelength or height difference, this implies good consistency between the two observation sensors.
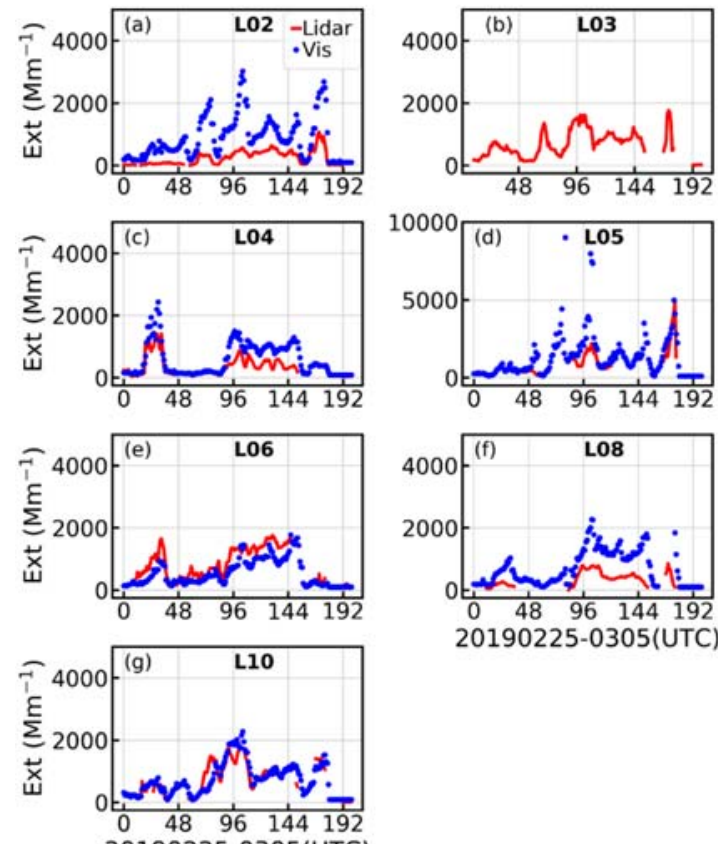

0190225-0305
Table 2. Statistical results about lidar and extinction coefficients on the ground. Positive bias means that visiometer observations higher than lidar observations. All the correlation coefficients have passed the t-test with confidence level 95\%.

\begin{tabular}{|c|c|c|}
\hline Lidar & Ext Bias $\left(\mathbf{M m}^{-1}\right)$ & $\mathbf{R}$ \\
\hline L02 & 661.48 & 0.82 \\
\hline L03 & $\backslash$ & $\backslash$ \\
\hline L04 & 291.52 & 0.84 \\
\hline L05 & 640.65 & 0.71 \\
\hline L06 & -274.53 & 0.91 \\
\hline L08 & 711.76 & 0.68 \\
\hline L10 & 24.15 & 0.87 \\
\hline Mean & 342.50 & 0.80 \\
\hline
\end{tabular}

\subsection{AOD}

AOD from Lidar observation and Himawari-8 satellite were compared with each other (Figure 5 and Table 3 ). Lidar AOD was higher than satellite AOD retrievals at most stations with the mean value 0.17 . Both sensors described the trend of pollution process at most stations. Two sensors had good consistency with the mean correlation coefficient value 0.46 . And L03 had the highest $\mathrm{R}$ value 0.56 .
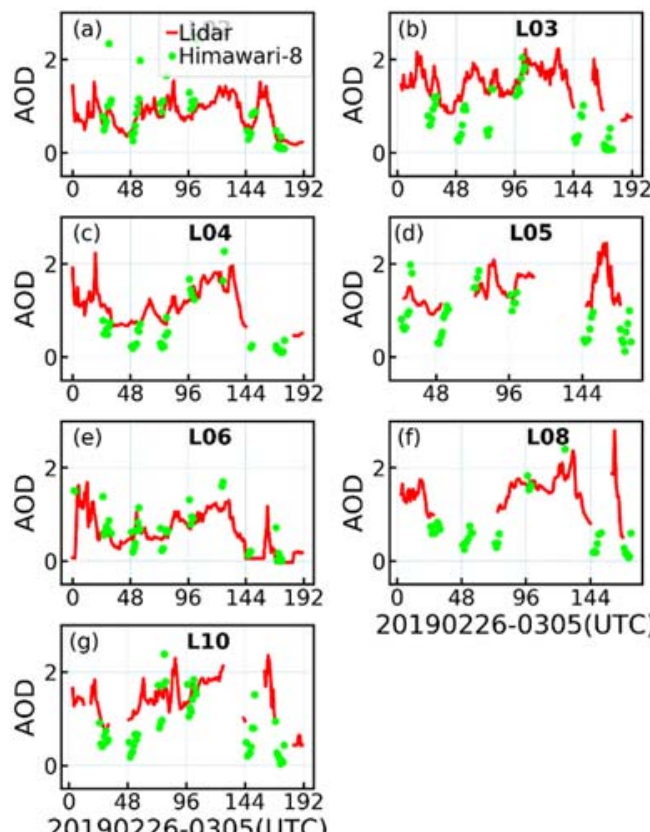

20190226-0305(UTC)

Fig. 5. AOD observed by Lidar (red line) and Himawari- 8 satellite (lime dot).

Table 3. Statistical results about AOD retrieved by Lidar and

Himawari-8 satellite. Positive bias means that satellite retrievals are higher than Lidar retrievals. All the correlation coefficients have passed the t-test with confidence level $95 \%$.

\begin{tabular}{|c|c|c|}
\hline Lidar & AOD Bias & R \\
\hline L02 & 0.14 & 0.40 \\
\hline L03 & -0.49 & 0.59 \\
\hline
\end{tabular}

Fig. 4. Aerosol extinction coefficient observed by visiometer on the ground (blue dot) and by Lidar (average value between 100$150 \mathrm{~m}$, red line). 


\begin{tabular}{|c|c|c|}
\hline L04 & -0.25 & 0.43 \\
\hline L05 & -0.31 & 0.51 \\
\hline L06 & 0.20 & 0.44 \\
\hline L08 & -0.17 & 0.41 \\
\hline L10 & -0.29 & 0.46 \\
\hline Mean & -0.17 & 0.46 \\
\hline
\end{tabular}

\subsection{Aerosol Extinction Profiles}

Aerosol extinction profiles at 7 Lidar stations were also compared with CALIPSO retrievals. Unfortunately, CALIPSO did not pass through Lidar station accurately during our study period. Near time (Figure 6 (a)) or near orbit (Figure 6 (b)) were adopted. The altitude of maximum aerosol extinction coefficient value retrieved by CALIPSO was at $4 \mathrm{~km}$ in Figure 6 (a). Both Lidar observed two peak values. They were located at the height of approximately $3 \mathrm{~km}$ and $1 \mathrm{~km}$. The altitude of maximum aerosol extinction coefficient value retrieved by CALIPSO was at $2 \mathrm{~km}$ in Figure 6 (b). Seven Lidar all have one maximum value. They were located at the height of less than $1 \mathrm{~km}$. From the analysis above, Lidar had similar vertical structures at a time. CALIPSO and Lidar had similar vertical structures in the troposphere. CALIPSO retrievals did not describe the aerosol vertical structure of the boundary layer during our study period. Of course, the time or location difference brought uncertainty to our analysis.
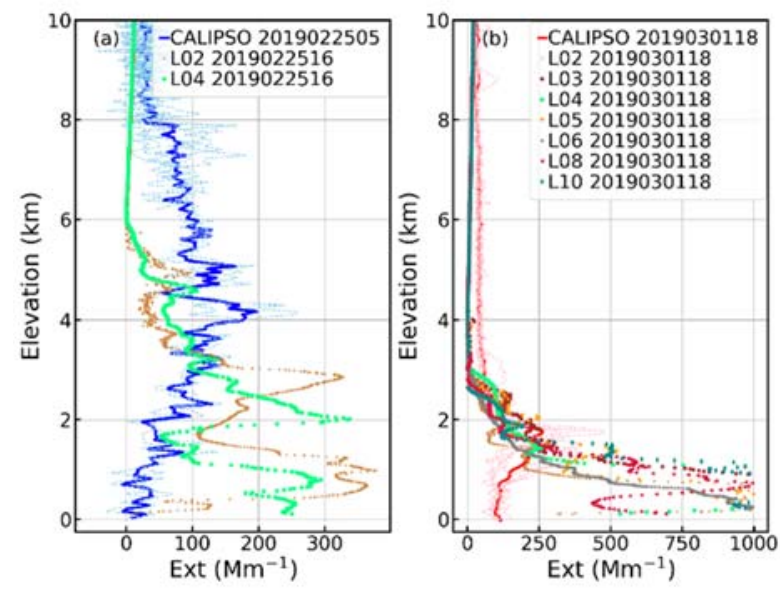

Fig. 6. Extinction coefficient profiles retrieved by CALIPSO (dashed line denotes signal profile, bold line denotes their average value) and Lidar (scatter).

\section{Conclusions and Discussions}

A fine aerosol particle pollution episode happened in mega city Beijing during February 25th - March 5th. Aerosol extinction coefficient profiles were observed at 7 Lidar stations. And cross comparing was conducted between different kind of sensors.

Different aerosol optical property instruments were used to verify Lidar retrievals. Ground visibility observations were used to verify near ground Lidar observations. High correlation coefficient was found (0.8) between visiometer and Lidar observations. Lidar's Column integrated aerosol extinction coefficient was also verified by Himawari-8 aerosol optical depth (AOD) data. The correlation coefficient between two AOD retrievals was 0.4 . And the mean bias was -0.17 . CALIPSO aerosol property profiles was also used to verify Lidar retrievals. Lidar retrievals was inconsistent with CALIPSO in the boundary layer.

It should be mentioned there are wavelength, height, time or location difference in the comparative data in this study. Other widely used Lidar equipment like Micro Pulse Lidar should be used in data verification. Overall, the Lidar observations during the megacity field experiment are acceptable for observation research and model validation.

\section{Acknowledgements}

This work was performed under the auspices of the National Natural Science Foundation of China (Grants 41705133), and the National Key Research and Development Program of China (2017YFC1501702).

\section{References}

1. Z. Qian, Y. Bao, F. Shen, M. Zhang, W. Tang, 2019, Marin. Forec. 36(6), 9-21(2019).

2. K. Bessho, K. Date, M. Hayashi, A. Ikeda, T. Imai, et al., J. Meteorol. Soc. of JPN. Ser.II94, 151-183 (2016).

3. H. Shang, L. Chen, H Letu, M. Zhao, S. Li, S. Bao, J. Geophys. Res.: Atmosphere 122(6), 3528-3543 (2017).

4. Powell, K. A. et al., J. Atmos. Oceanic Technol. 26, 2015-2033 (2009).

5. Vernier, J.-P. et al., J. Geophys. Res., 114, D00H10 (2019).

6. D. Wu, Z. Wang, B. Wang, J. Zhou, Y. Wang, Appl. Phys. B, 102, 185-195 (2010).

7. J. Shen, N. Cao, N., Atmos. Environ. 117767 (2020).

8. H. Koschmieder, Mon. Wea. Rev. 58, 439-444 (1930).

9. L. Pei, Z. Yan, Z. Sun, S. Miao, Y. Yao, 2018. Atmos. Chem. Phys., 18, 3173-3183 (2018).

10. L. Gao, L. Cao, Y. Zhang, P. Yan, J. Jing, et al. Sci. Tot. Env. 732, 138905 (2020). 